\title{
TOTALLY ORDERED $D$-CLASS DECOMPOSITIONS
}

BY K. H. HOFMANN AND P. S. MOSTERT ${ }^{1}$

Communicated by E. Hewitt, June 8, 1964

Introduction. An irreducible semigroup is a compact connected semigroup with identity which contains no proper compact connected subsemigroup containing the identity and meeting the minimal ideal. Since every compact connected semigroup with identity has an irreducible subsemigroup joining its identity to its minimal ideal, the study of the category of irreducible semigroups is basic in any quest for information about the structure of compact semigroups with identity. In a previous announcement [4], the authors described the notion of a hormos and conjectured that an irreducible semigroup is an irreducible hormos. (The structure of the latter was completely described-without proofs-in that announcement.) We also announced a number of categories of semigroups for which we are able to obtain this result.

One distinguishing feature of an irreducible hormos is that the $D$ class (and in fact even the $H$-class) decomposition is a totally ordered semigroup-in fact an $I$-semigroup [6]. It may be conjectured that a study of semigroups whose $D$-class decomposition is totally ordered might lead to a description of all irreducible semigroups, and it is this motive which brings us to the results of this announcement.

We shall give a brief outline of the techniques we use to prove our results. The details will appear in our forthcoming book. For terminology, the reader is referred to [1] and [4].

1. Admissible spaces. The following concept is of mere technical interest but it is needed for the principal results of this paper. A space $X$ will be called admissible if every compact subspace $Y$ of $X$ for which there is a totally ordered compact connected space $T$ and function

$$
F: Y \times T \times Y \rightarrow Y
$$

with the following properties is necessarily a point:

(a) $F(F(x, t, y), s, y)=F(x, t \wedge s, y)$ for all $x, y \in Y, t, s \in T$ where $t \wedge s=\min \{t, s\}$;

${ }_{1}^{1}$ This work was partially supported by NSF Grant GP 1877. 
(b) $F(F(x, t, y), t, z)=F(x, t, z)$ for all $x, y, z \in Y, t \in T$;

(c) $F(x, 1, y)=x, F(x, 0, y)=y$ for all $x, y \in Y$, where 0 and $1 \mathrm{de}$ note the minimum and maximum elements of $T$, respectively;

(d) for each $y \in Y, F(\cdot, \cdot, y)$ is continuous.

Proposition 1. If $X$ is a finite-dimensional compact space, then $X$ is admissible. ${ }^{2}$

The proof of this result uses the Vietoris mapping theorem on a carefully constructed subspace.

There is an example, due to John Stallings, of an infinite-dimensional nonadmissible compact connected space.

2. Clifford semigroups. A Clifford semigroup is a semigroup which is a union of groups. The algebraic structure has been to a large extent determined by Clifford [2]. (See also [1, p. 122].) Let $S$ be a compact Clifford semigroup. (It is not assumed that $S$ has an identity.) It is known that the $D$-class decomposition, which we denote by $T$, is a semilattice in this case, and that the natural projection $\pi: S \rightarrow T$ is a homomorphism. If we assume $T$ is totally ordered by the natural ordering of the $D$-classes (i.e., $D(x) \leqq D(y)$ if $S x S \subset S y S),{ }^{3}$ then $T$ is a totally ordered semilattice. We denote by 0 the minimal element and 1 the maximal element of $T$. We shall say that $S$ has connecting homomorphisms if for each "gap" $[a, b]$ in $T$ (if the open interval ]$a, b[$ is empty, $[a, b]$ is called a $g a p)$, there is a homomorphism $f: \pi^{-1}(b) \rightarrow \pi^{-1}(a)$ such that all idempotents of $\pi^{-1}(a)$ which are below an idempotent of $\pi^{-1}(b)$ are in the image under $f$. (Notice in particular that if $T$ is connected, $S$ trivially has connecting homomorphisms.)

Proposition 2. Let $S$ be a compact Clifford semigroup whose D-class decomposition is totally ordered and which has connecting homomorphisms. If the set $E$ of idempotents is admissible, then there is a homomorphism

$$
\psi: \pi^{-1}(1) \times S / D \rightarrow S
$$

with the following properties:

(i) $\psi(a, 1)=a$ for each $a \in \pi^{-1}(1)$;

(ii) the following diagram commutes

2 The dimension we use is the Cech cohomology dimension [3].

${ }^{3}$ In the semigroups we consider, it is the case that $\{x\} \cup_{S x} \cup_{x S C} S_{x S}$. Hence, the $D-\left(=J_{-}\right)$class decomposition is determined by $x \equiv y D$ if and only if $S x S=S y S$. 


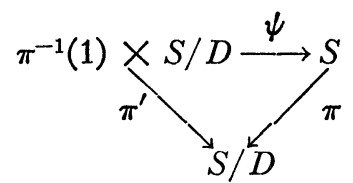

where $\pi^{\prime}$ is the natural projection. In general, $\psi$ is not surjective.

From the fact that $E$ is admissible, one is enabled to prove that through each idempotent $e \in S$, there is one and only one totally ordered compact semigroup $T_{e}$ such that $\pi \mid T_{e}$ is an isomorphism onto $[0, \pi(e)]$. This then leads to the proposition after a series of more or less technical-though not particularly deep-lemmas. Nevertheless, we would rank the proof of this result and its application to the proof of Theorem 1 as a breakthrough of nontrivial proportions. In particular, it sheds a great deal of light on the location of Koch's arcs ${ }^{4}$ in semigroups.

THEOREM 1. Let $S$ be a compact Clifford semigroup with identity such that $S / D$ is connected and suppose that the set of idempotents is admissible. Then every I-semigroup containing the identity is in the centralizer of the maximal subgroup of the identity. At least one such exists joining the identity to the minimal ideal, and no two such determine the same collection of D-classes.

REMARK. In particular, if $S$ is a compact Clifford semigroup such that $S / D$ is connected and totally ordered, and such that the set of idempotents is admissible, then, through each idempotent of the maximal $D$-class, there is precisely one Koch's arc. This is no longer true in the absence of admissibility.

3. Semigroups over "gaps." Again in our study of unital semigroups whose $D$-class decomposition is totally ordered, we are forced to consider semigroups without identity. This time they arise from looking at a "gap" between regular $D$-classes in the ordering, and here we are able to obtain much better information. However, because of our inability to handle the situation where an arc of regular $D$-classes occurs, we are unable to apply the full power of this theorem. (Recall that a regular $D$-class is one which contains an idempotent [1].)

Let $S$ be a compact semigroup with identity whose $D$-class decomposition is totally ordered. Let $X$ and $Y$ be compact spaces and $[]:, Y \times X \rightarrow S$ be a continuous function. Suppose, further, that

${ }^{4}$ A Koch's arc is an idempotent $I$-semigroup. It was R. J. Koch [5] who introduced the technique of obtaining such semigroups in compact semigroups. 
there are distinguished points $a \in X$, and $b \in Y$ such that $[b, a]=1$. Then the Rees product [8] $R=[X, S, Y]$ with respect to the sandwich function $[$,$] and multiplication (x, s, y)\left(x^{\prime}, s^{\prime}, y^{\prime}\right)$ $=\left(x, s\left[y, x^{\prime}\right] s^{\prime}, y\right)$ admits a homomorphism $\phi: S \rightarrow R$, and an orderpreserving one-one map $\phi^{\prime}: S / D \rightarrow R / D$ such that the following diagram commutes:

$$
\begin{array}{cc}
S & \stackrel{\phi}{\rightarrow} R \\
\pi \downarrow & \quad \phi^{\prime} \quad \downarrow \pi^{\prime} \\
S / D & \stackrel{\rightarrow}{\rightarrow} R / D .
\end{array}
$$

The morphism $\phi$ is defined by $\phi(s)=(a, s, b)$. We shall denote the subspace $A \times T \times B$ of $R$, where $A \subset X, T \subset S, B \subset Y$, by $[A, T, B]$.

THEOREM 3. Let $S$ be a compact semigroup with connected totally ordered D-class decomposition. Suppose that the maximal D-class contains an idempotent $e$, and that $D_{1}<D(e)$ is a regular $D$-class, but there is no regular $D$-class between $D_{1}$ and $D(e)$. Then there exist

(1) a completely irreducible semigroup $\Sigma$ in the centralizer of $H(e)$, and $e$ is its identity;

(2) compact spaces $X$ and $Y$ with distinguished points $a$ and $b, r e-$ spectively;

(3) a map $[]:, Y \times X \rightarrow e$ Se such that $[b, a]=e$;

(4) a homomorphism $\phi:[X, e S e, Y] \rightarrow S$, such that

(i) $S_{1}=S-U\left\{D(x) \leqq D_{1}\right\} \subset \phi([X, \Sigma, Y])$,

(ii) if $\pi_{1}: \Sigma \rightarrow \Sigma / H, \pi: S \rightarrow S / D$ are the natural projections, then there is an order-preserving map $m: \Sigma / H \rightarrow S / D$ such that the diagram

$$
\begin{array}{cc}
\lfloor X, \Sigma, Y] \stackrel{\phi}{\longrightarrow} S \\
\downarrow(x, s, y) \rightarrow \pi_{1}(s) \quad \begin{array}{r}
\downarrow \pi \\
\Sigma / H \longrightarrow
\end{array} / D
\end{array}
$$

commutes, providing $D(e)$ and $D(1)$ are subsemigroups. ${ }^{5}$

Further, if $D_{1}$ is a subsemigroup, then these are equivalent:

(a) $\phi([X, \Sigma, Y])$ is a semigroup and $\pi \mid \operatorname{im} \phi$ is a homomorphism,

(b) $[Y, X] \subset H(e)$,

(c) $D(e)$ is a subsemigroup.

Moreover, when these conditions are satisfied, $\phi[X, \Sigma, Y]=S_{1}^{*}$ (the closure of $S_{1}$ ).

$\sqrt{5} \Sigma / H$ denotes $\Sigma$ modulo its $H$-class relation. 
The proof of this theorem follows from a sequence of rather technical, but not particularly deep, lemmas once we have the following powerful result:

THEOREM 2. Let $S$ be a compact semigroup with identity and maximal subgroup $H$. Suppose that there is a neighborhood of the identity without idempotents other than 1. If the orbit space of $H \times H$ acting on $S$ under $((h, k), y) \rightarrow h y k^{-1}$ is an arc in some neighborhood of $H \cdot 1 \cdot H=H$, then there is a one-parameter semigroup $f:[0, \infty) \rightarrow S$ such that $f(t) \in H$ if and only if $t=0$, and such that the image of $f$ is in the centralizer of $H$ (i.e., $f(t) h=h f(t)$ for all $t \in[0, \infty)$ and $h \in H$ ).

This theorem is actually contained in Theorem $\mathrm{E}$ which we announced in [4]. Its proof uses the structure theory of compact groups and their homological properties, the construction theory for one-parameter semigroups, and an involved theory of peripherality which the authors developed for this purpose. Even a brief outline of its proof could not be given in the short space of a few pages.

4. The main theorem. Since the stating of the main theorem for a larger class of semigroups than those with identity requires little additional effort, we give it in the following form. Because it is a rather complete structure theorem, the conclusions become a bit involved. The reader may glance at the list of corollaries, all of which follow with almost no effort from the main theorem, to get some idea of its power.

MAIN THEOREM. Let $S$ be a compact semigroup satisfying the following conditions:

(a) $S^{2}=S$;

(b) the union of all subgroups of $S$ forms a semigroup;

(c) $S / D$ is connected and totally ordered relative to the natural order $D(x) \leqq D(y)$ if $S x S \subset S y S$;

(d) in each D-class, the components of the set of idempotents are admissible spaces;

then the following properties hold for $S$ :

A. The relation $D$ is a congruence, the $D$-class projection $\pi: S \rightarrow S / D$ is a homomorphism, and $S / D$ is an I-semigroup.

B. There are subsemigroups $S_{r}$ and $S_{l}$ such that

(i) $\pi\left(S_{l}\right)=\pi\left(S_{r}\right)=S / D$;

(ii) if $a^{2}=a \in S / D$, then $\pi^{-1}(a) \cap S_{l}$ (resp. $\left.\pi^{-1}(a) \cap S_{r}\right)$ is a direct product of a group and a right- (resp. left-) zero semigroup;

(iii) $S=S_{l} S_{r}$;

(iv) $S_{r} \cap S_{l}=T$, and $T$ is a hormos such that $\pi(T)=S / D$. 
C. If $a^{2}=a \in S / D$, then the semigroup $\pi^{-1}(a)$ is a completely simple semigroup.

D. If $[a, b]$ is an interval in $S / D$ which is an I-semigroup with only the two idempotents $a$ and $b$, then there is a compact connected abelian group $A$ and a homomorphism

$$
f:] a, b] \rightarrow A
$$

onto a dense one-parameter semigroup and a homomorphism $\phi$ mapping $S_{a b}$ onto $\left.\left.\left(\pi^{-1}(] a, b\right]\right)\right)^{*}$, where

$$
\left.\left.S_{a b}=\pi^{-1}(b) \times(\{(r, f(r)): r \in] a, b]\right\} \cup(\{a\} \times A)\right),
$$

such that

$$
\begin{gathered}
\underset{\pi^{\prime} \searrow}{S_{a b}} \stackrel{\phi}{\longrightarrow} \pi^{-1}([a, b]) \\
\\
{[a, b]}
\end{gathered}
$$

is a commutative diagram, where $\pi^{\prime}(x, y, z)=y$.

E. If $[a, b]$ is an idempotent semigroup, then $\pi^{-1}([a, b])$ is a Clifford semigroup and there is a homomorphism $\phi$ of the semigroup $S_{a b}$ into $\pi^{-1}([a, b])$, where

$$
S_{a b}=\pi^{-1}(b) \times[a, b]
$$

such that

$$
\begin{aligned}
& \underbrace{S_{a b} \stackrel{\phi}{\longrightarrow} \pi^{-1} / \pi}_{\pi^{\prime} \searrow}([a, b]) \\
& {[a, b]}
\end{aligned}
$$

is a commutative diagram with $\pi^{\prime}(x, y)=y$.

F. If $a^{2}=a, b^{2}=b \in S / D, a<b$, then there is a retraction homomorphism $\phi$ mapping $\pi^{-1}([a, b])$ into $\pi^{-1}(a)$ such that there is precisely one idempotent in $\phi\left(\pi^{-1}(b)\right)$ which is below a given idempotent in $\pi^{-1}(b)$ in the natural partial order on $E$.

Conversely, if A is satisfied, then (a), (b) and (c) are true.

The proof involves our knowledge of the Clifford semigroups with connecting homomorphisms and the structure of the "semigroups over gaps" and in turn their structure as homomorphic images of a product of a $D$-simple semigroup and a "sigma" semigroup $\Sigma(f, A, a)$. 
The semigroup $T$ is obtained uniquely and $S_{r}$ and $S_{l}$ defined as $S_{r}=\bigcup\{R(t): t \in T\}, S_{l}=\bigcup\{L(t): t \in T\}$.

COROLlaRy 1. If $S$ is a compact connected semigroup with identity and the D-class decomposition is a congruence, then $S$ contains an irreducible hormos in the centralizer of the maximal subgroup joining the identity to the minimal ideal providing the set of idempotents is an admissible space. (In particular, if the set of idempotents is finite dimensional, then the statement is true.)

COROLlARY 2. If $S$ is a compact connected semigroup with identity such that $x S \subset S x$ for every $x$, and the set of idempotents is admissible, then $S$ contains an irreducible hormos in the centralizer of the maximal subgroup and joining the identity to the minimal ideal.

REMARK. Rothman [7] has studied the case when the L-class decomposition of a compact connected semigroup is totally ordered under the natural order. Since, as he has shown, this implies that $S^{2}=S$ and the $L-\left(=D_{-}\right)$class decomposition is a congruence, the main theorem gives a rather complete picture of this case when the set of idempotents satisfies the admissibility condition stated there. (When $x S=S x$ for every $x$, the result holds without these conditions [4].)

Corollary 3. If $S$ is an irreducible semigroup whose D-class decomposition is totally ordered and such that the union of its maximal subgroups is a semigroup, then $S$ is an abelian hormos, provided also that the set of idempotents is admissible (or finite dimensional).

\section{BIBLIOGRAPHY}

1. A. H. Clifford and G. B. Preston, The algebraic theory of semigroups, Math. Surveys No. 7, Amer. Math. Soc., Providence, R.I., 1961.

2. A. H. Clifford, Semigroups admitting relative inverses, Ann. of Math. (2) 42 (1941), 1037-1049.

3. H. Cohen, $A$ cohomological definition of dimension for locally compact Hausdorff spaces, Duke Math. J. 21 (1954), 209-224.

4. K. H. Hofmann and P. S. Mostert, Irreducible semigroups, Bull. Amer. Math. Soc. 70 (1964), 621-627.

5. R. J. Koch, Arcs in partially ordered spaces, Pacific J. Math. 9 (1959), 723-728.

6. P. S. Mostert and A. L. Shields, On the structure of semigroups on a compact manifold with boundary, Ann. of Math. (2) 65 (1957), 117-143.

7. N. J. Rothman, Linearly quasi-ordered semigroups, Proc. Amer. Math. Soc. 13 (1962), 352-357.

8. A. D. Wallace, The Rees-Suschkewitsch structure theorem for compact simple semigroups, Proc. Nat. Acad. Sci. U.S.A. 42 (1956), 430-432.

The Tulane University of Louisiana 